\title{
PRESENTATION OF ASSOCIATED GRADED RINGS OF COHEN-MACAULAY LOCAL RINGS
}

\author{
YOUNG-HYUN CHO
}

\begin{abstract}
Let $(R, m)$ be a local ring and $I$ be an m-primary ideal such that $\operatorname{dim}_{k}(I / I \mathrm{~m})=I$, where $k=R / \mathrm{m}$. Denote the associated graded ring with respect to $I, \bigoplus_{n=0}^{\infty} I^{n} / I^{n+1}$, by $G_{I}(R)$. Then $G_{I}(R) \simeq R / I\left[X_{1}, \ldots, X_{l}\right] / \mathcal{L}$, for some homogeneous ideal $\mathrm{L}$. Set $M=\max \operatorname{deg}_{1 \leqslant i \leqslant t} f_{i}$, where $\left\{f_{1}, \ldots, f_{t}\right\}$ is a set of homogeneous elements which form a minimal basis of $\mathcal{L}$. The main result in this note is that if $R$ is a Cohen-Macaulay local ring of dimension 1 and if $G_{I}(R)$ is free over $R / I$, then $M \leqslant r(I)+1$, where $r(I)$ is the reduction number of $I$. It follows that $M \leqslant e(R)$ where $e(R)$ is the multiplicity of $R$.
\end{abstract}

1. Introduction. First of all, we refer the reader to [5] for notations. Let $(R, \mathrm{~m})$ be a local ring and $I$ be an m-primary ideal such that $\operatorname{dim}_{k}(I / I \mathrm{~m})=l$, where $k=R / \mathrm{m}$. Choose a minimal basis of $I, x_{1}, \ldots, x_{l}$, and give a map $\phi$ : $R / I\left[X_{1}, \ldots, X_{l}\right] \rightarrow G_{I}(R)$ defined by $\phi\left(X_{i}\right)=\bar{x}_{i}$, for all $i=1, \ldots, l$. Here, $\bar{x}_{i}$ denotes the image of $x_{i}$ in $I / I^{2}$. Then we can see that $G_{I}(R)$ is the homomorphic image of

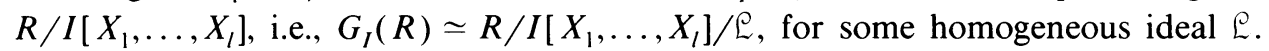
When $G_{I}(R)$ is free over $R / I$, $巳$ does not depend on the choice of the basis of $I$, because if we have another minimal basis of $I, y_{1}, \ldots, y_{l}$, and give a map $\phi^{\prime}$ : $R / I\left[X_{1}, \ldots, X_{l}\right] \rightarrow G_{I}(R)$ defined by $\phi^{\prime}\left(X_{i}\right)=\bar{y}_{i}$, for all $i=1, \ldots, l$, then we get the following commutative diagram

$$
\begin{aligned}
& 0 \rightarrow \operatorname{Ker} \phi \quad \rightarrow \quad R / I\left[X_{1}, \ldots, X_{l}\right] \stackrel{\phi}{\rightarrow} G_{I}(R) \rightarrow \rightarrow 0 \\
& \downarrow i \quad \downarrow=\quad \downarrow \psi \\
& 0 \rightarrow \operatorname{Ker} \phi^{\prime} \rightarrow R / I\left[X_{1}, \ldots, X_{l}\right] \stackrel{\phi^{\prime}}{\rightarrow} G_{I}(R) \rightarrow 0 \\
& \psi\left(\bar{x}_{i}\right)=\bar{y}_{i}, \quad \text { for } i=1, \ldots, l,
\end{aligned}
$$

and this induces an isomorphism $i: \operatorname{Ker} \stackrel{\sim}{\rightarrow} \operatorname{Ker} \phi^{\prime}$. Let $\left\{f_{1}, \ldots, f_{t}\right\}$ be a set of homogeneous elements which form a minimal basis of $E$. We will study the upper bound max $\operatorname{deg}_{1 \leqslant i \leqslant t} f_{i}=M$.

REMARK 1. We can see that the number of elements and the set of degrees of

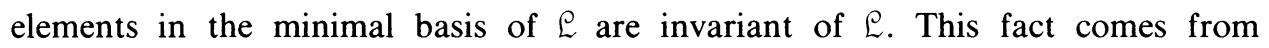
[1, III-§1, Definition 1 and Lemma 1] with a slight modification.

Received by the editors September 15, 1982.

1980 Mathematics Subject Classification. Primary 13H10, 13H15.

${ }^{1}$ The author was partially supported by the Korea Science and Engineering Foundation research grant. 
2. The case when $\operatorname{dim} R=1$. We consider the case when $R$ is a Cohen-Macaulay local ring of dimension 1 . Before we prove the main theorem, we will mention the flat change of local rings $R \rightarrow R(u)=R[u]_{\mathrm{m} R[u]}$, where $u$ is an indeterminate.

Lemma 2. Let $(R, \mathrm{~m})$ be a local ring. Consider $R \rightarrow R(u)$. Then

(a) $R(u)$ is faithfully flat over $R$.

(a) $I R(u) \cap R=I$, for any ideal $I$ in $R$.

(b) $\operatorname{dim} R=\operatorname{dim} R(u)$.

(c) depth $R=\operatorname{depth} R(u)$.

(d) $v(I)=v(I R(u))$, for any ideal $I$ in $R$.

(e) $\lambda(R / I)=\lambda(R(u) / I R(u))$, for any m-primary ideal $I$.

(f) $e(I)=e(I R(u))$, for any m-primary ideal $I$.

Proof. (a) and (c) See [3, p. 27, Corollary and (21c)].

(b) By [3, Theorem 19(2)], ht $(\mathrm{m} R(u))=\mathrm{ht}(\mathrm{m} R(u) \cap R)=\mathrm{ht}(\mathrm{m})$.

(d) $v(I)=\operatorname{dim}_{k}(I / I \mathrm{~m})$, where

$k=R / \mathrm{m}=\operatorname{dim}_{k(u)}(I / I \mathrm{~m} \otimes R(u))=\operatorname{dim}_{k(u)}(I R(u) / I \mathrm{~m} R(u))=v(I R(u))$.

(e) If $0=I / I \subset I_{1} / I \subset \cdots \subset R / I$ is a composition series of $R / I$, then $0=$ $\operatorname{IR}(u) / \operatorname{IR}(u) \subset I_{1} R(u) / I R(u) \subset \cdots \subset R(u) / I R(u)$ is also.

(f) From (e), $\lambda\left(I^{n} / I^{n+1}\right)=\lambda\left(I^{n} R(u) / I^{n+1} R(u)\right)$, for any $n>0$. Thus $e(I)=$ $e(\operatorname{IR}(u))$ follows.

LEMMA 3. For any ideal $I$ in a local ring $(R, \mathrm{~m})$,

(a) $\operatorname{dim} G_{I}(R)=\operatorname{dim} G_{I R(u)}(R(u))$.

(b) depth $G_{I}(R)=\operatorname{depth} G_{I R(u)}(R(u))$. Here, $\operatorname{depth} G_{I}(R)\left(\right.$ or $\left.\operatorname{depth} G_{I R(u)}(R(u))\right)$ means a maximal $G_{I}(R)$-sequence $\left(\right.$ or $G_{I R(u)}(R(u))$-sequence) in $\mathrm{m} / I \oplus I / I^{2} \oplus \cdots$ ( or $\left.\mathrm{m} R(u) / I R(u) \oplus I R(u) / I^{2} R(u) \oplus \cdots\right)$ ).

Proof. (a) Consider the Rees ring $\mathscr{G}=R[I t]\left[t^{-1}\right]$ of $R$ with respect to $I$. Then $t^{-1}$ is not a zero divisor in $\mathscr{R}$ and $\operatorname{dim} \mathscr{R}=\operatorname{dim} R+1$. (See [4, Remark 3.7].) Since $G_{I}(R)=\stackrel{6}{\Omega} / t^{-1} \Re, \operatorname{dim} G_{I}(R)=\operatorname{dim} R$. By the same way, $\operatorname{dim} G_{I R(u)}(R(u))=$ $\operatorname{dim} R(u)$. By Lemma $2(\mathrm{~b})$, the assertion follows.

(b) Put $\mathfrak{N}=\mathrm{m} / I \oplus I / I^{2} \oplus \cdots$ and $\mathfrak{N}(u)=\mathfrak{m} R(u) / I R(u) \oplus I R(u) / I^{2} R(u)$ $\oplus \cdots$. We only need to show that depth $G_{I}(R)_{\Re}=\operatorname{depth} G_{I R(u)}(R(u))_{\mathscr{N}(u)}$. Since $R(u)$ is faithfully flat over $R$, by [3,(4a)], $R(u) \otimes_{R} G_{I}(R)=G_{I R(u)}(R(u))$ is faithfully flat over $G_{I}(R)$. Then by [3,p. 154, Corollary],

$$
\operatorname{depth} G_{I}(R)_{\mathcal{O R}}=\operatorname{depth} G_{I R(u)}(R(u))_{\mathcal{O R}(u)} .
$$

Now we are going to prove the main theorem. The reduction number of $I$, denoted by $r(I)$, means the least integer $r$ such that $x I^{r}=I^{r+1}$ for some $x \in I \backslash I^{2}$.

THEOREM 1. Let $(R, \mathrm{~m})$ be a 1-dimensional Cohen-Macaulay local ring and $I$ be an m-primary ideal such that $G_{I}(R)$ is free over $R / I$. Then $M \leqslant r(I)+1$.

Proof. Set $\operatorname{dim}_{k}(I / I \mathrm{~m})=l$, where $R / \mathrm{m}=k$, and let $\phi$ map $R / I\left[X_{1}, \ldots, X_{l}\right]$ onto $G_{I}(R)$ by $\phi\left(X_{i}\right)=\bar{x}_{i}$, where $x_{1}, \ldots, x_{l}$ is a minimal basis of $I$. Let $\mathcal{L}=$ Kernel $\phi$ 
and let $f_{1}, \ldots, f_{t}$ be any minimal basis of $E$ consisting of homogeneous elements. Then

$$
G_{I R(u)}(R(u)) \simeq R(u) / I R(u)\left[X_{1}, \ldots, X_{l}\right] /\left(f_{1}, \ldots, f_{t}\right) \otimes R(u)
$$

and $f_{1}, \ldots, f_{t}$ is a minimal set of generators for (Kernel $\left.\phi\right) \otimes R(u)$. The degree of $f_{i}$ in $R / I\left[X_{1}, \ldots, X_{l}\right]$ is same as the degree of $f_{i}$ in $R(u) / I R(u)\left[X_{1}, \ldots, X_{l}\right]$ by Lemma 2 . Hence, by Remark 1 and Lemmas 2 and 3 we can show that theorem just in the case of the ring $R(u)$.

Therefore we can assume $R / \mathrm{m}$ is infinite and we can pick up $x_{1}$ which is a superficial element of $I$ such that $x_{1} I^{r}=I^{r+1}$, where $r=r(I)$. (See [6, Lemma 5, p. 286] for the existence of $x_{1}$.) We can check that $\left(0: \bar{x}_{1} G_{I}(R)\right) \cap I^{n} / I^{n+1}=0$ for all $n \geqslant r-1$. Choose $x_{2}, \ldots, x_{l}$ such that $\left\{\bar{x}_{i}\right\}_{i=1, \ldots l}$ is a minimal basis for $I / I^{2}$, and define $\phi: R / I\left[X_{1}, \ldots, X_{l}\right] \rightarrow G_{I}(R)$ by $\phi\left(X_{i}\right)=\bar{x}_{i}$ as before. Let $\mathcal{E}=$ Kernel $\phi$. Then $G_{I}(R) \simeq R / I\left[X_{1}, \ldots, X_{l}\right] / \mathcal{L}$. Since $x_{1} I^{r}=I^{r+1}$,

$$
\bar{x}_{1}\left(I / I^{2} \oplus I^{2} / I^{3} \oplus \cdots\right)^{r}=\left(I / I^{2} \oplus I^{2} / I^{3} \oplus \cdots\right)^{r+1},
$$

i.e., $X_{1}\left(X_{1}, \ldots, X_{l}\right)^{r}+\varrho / \varrho=\left(X_{1}, \ldots, X_{l}\right)^{r+1}+\varrho / \varrho$. Hence $\left(X_{1}, \ldots, X_{l}\right)^{r+1}+\varrho=$ $X_{1}\left(X_{1}, \ldots, X_{l}\right)^{r}+\varrho$, therefore $\left(X_{1}, \ldots, X_{l}\right)^{r+1} \subset X_{1}\left(X_{1}, \ldots, X_{l}\right)^{r}+\varrho$. For convenience, let $P$ denote $\left(X_{1}, \ldots, X_{l}\right)$ in following Claims 1 and 2.

Claim 1. $巳 \cap X_{1}^{j+1} P^{r} \subset P^{j} \mathcal{L}$, for any $j \geqslant 0$.

Proof. We will prove by induction on $j$. When $j=0, \varrho \cap X_{1} P^{r} \subset \varrho$ is obviously true. Suppose $j>0$ and the claim is true for $j-1$. Let $X_{1}^{j+1} g \in \mathcal{L}$, where $g \in P^{r}$. We write $g=\sum_{i=r}^{p} g_{i}$ with $g_{i}$ homogeneous and degree of $g_{i}=i$, for any $i=r, \ldots, p$. Since $\sum_{i=r}^{p} X_{1}^{j+1} g_{i} \in \mathcal{E}$ and $\mathcal{E}$ is a homogeneous ideal, $X_{1}^{j+1} g_{i} \in \mathcal{E}$ for $i=r, \ldots, p$.

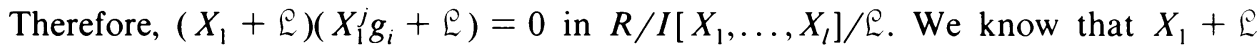
corresponds to $\bar{X}_{1}$ and $X_{1}^{j} g_{i}+\mathcal{L}$ corresponds to some elements in $I^{n} / I^{n+1}$, where $n \geqslant r+j$. Hence $X_{1}^{j} g_{i}+\mathcal{L}=0$ since $\left(0: \bar{x}_{1} G_{I}(R)\right) \cap I^{n} / I^{n+1}=0$ for all $n \geqslant r-1$. This means $X_{1}^{j} g_{i} \in \mathcal{L}$ for $i=r, \ldots, p$. By induction hypothesis, $X_{1}^{j} g_{i} \in P^{j-1} \varrho$, for $i=r, \ldots, p$. Now, $X_{1}^{j+1} g=X_{1} \sum_{i=r}^{p} X_{1}^{j} g_{i} \in P^{j} \varrho$. So we get $\varrho \cap X_{1}^{j+1} P^{r} \subset P^{j} \varrho$.

Claim 2. $\& \cap P^{r+1+j} \subset P^{j} \varrho$, for any $j \geqslant 0$.

Proof. Before Claim 1, we had $P^{r+1} \subset X_{1} P^{r}+$ L. Now

$$
\begin{aligned}
& P^{r+2}=P \cdot P^{r+1} \subset P\left(X_{1} P^{r}+\varrho\right) \\
& =X_{1} P^{r+1}+P \varrho \subset X_{1}\left(X_{1} P^{r}+\varrho\right)+P \varrho=X_{1}^{2} P^{r}+P \varrho .
\end{aligned}
$$

By the same way we get $P^{r+1+j} \subset X_{1}^{j+1} P^{r}+P^{j} \varrho$, for any $j \geqslant 0$. Next,

$$
P^{r+1+j} \cap \mathcal{L} \subset\left(X_{1}^{j+1} P^{r}+P^{j} \mathcal{L}\right) \cap \mathcal{L}=X_{1}^{j+1} P^{r} \cap \mathcal{L}+P^{j} \varrho \subset P^{j} \mathcal{L},
$$

since Claim 1 holds.

To finish the proof, let $\left\{f_{1}, \ldots, f_{t}\right\}$ be a set of homogeneous elements which form a minimal basis of $\mathcal{L}$. Assume some $f_{i}^{\prime}$ s have degree greater than $r+1$, say $\operatorname{deg} f_{1}=r$ $+1+j$, for some $j>0$. Then $f_{1} \in \varrho \cap\left(X_{1}, \ldots, X_{l}\right)^{r+1+j} \subset\left(X_{1}, \ldots, X_{l}\right)^{j \varrho}$. Therefore $f_{1}=\sum_{i=1}^{n} h_{i} g_{i}$ with $h_{i} \in\left(X_{1}, \ldots, X_{l}\right)^{j}, g_{i} \in \mathcal{L}$ and $h_{i}, g_{i}$ are homogeneous elements for any $i$. Since $f_{1}$ is a homogeneous element, we can assume $\operatorname{deg} f_{1}=\operatorname{deg} h_{i} g_{i}$ for any $i$. Now $\operatorname{deg} h_{i} g_{i}=r+1+j$ and $\operatorname{deg} h_{i} \geqslant j$ imply $\operatorname{deg} g_{i} \leqslant r+1$ so that $g_{i} \in \mathcal{E}$ is in $\left(f_{2}, \ldots, f_{t}\right)$. So, $f_{1} \in\left(f_{2}, \ldots, f_{t}\right)$. A contradiction follows from the minimality of the $f_{i}$ 's. 
Corollary 1 . Let $R, \mathrm{~m}, I$ be the same as in Theorem 1 . Then $M \leqslant e(R)$.

Proof. If $R$ is a discrete valuation ring, $G_{I}(R)=R / I[X]$, since $I=(x)$ for some regular element $x$. Otherwise $r(I) \leqslant e(R)-1$ (cf. Remark 1.5 and Corollary 2.2 of [2]). Hence $M \leqslant r(I)+1 \leqslant e(R)$. Of course the hypothesis that $G_{I}(R)$ is free over $R / I$ is satisfied by $I=m$.

3. The general case. For the proof of Theorem 2, we need the following lemma and corollary.

Lemma 4. Let $A$ be any ring and $L$ any homogeneous ideal in $A\left[X_{1}, \ldots, X_{n}\right]$. Then $f_{1}, \ldots, f_{l}$ generate $E$ if and only if $f_{1}+y_{L}, \ldots, f_{l}+\dot{L}$ generate $E / G \dot{L}$ over $A\left[X_{1}, \ldots, X_{n}\right] / \mathbb{L}$, where $\mathrm{g}$ is a homogeneous ideal contained in $\left(X_{1}, \ldots, X_{n}\right)$.

Proof. $(\rightarrow)$ It is obvious.

$(\leftarrow)$ Suppose $f_{1}, \ldots, f_{l}$ are elements in $E^{\prime}$ such that $\left(f_{1}, \ldots, f_{l}\right)+g E=E$. Then we get $\left(f_{1}, \ldots, f_{l}\right)+y_{L}=E$ for any $n$. If we choose any homogeneous element $g$ in $L$ with $\operatorname{deg} g=m$, then $g \in\left(f_{1}, \ldots, f_{l}\right)+g^{m+1}$. By comparing degrees, $g$ must belong to $\left(f_{1}, \ldots f_{l}\right)$. Therefore $\mathbb{L}^{\prime}=\left(f_{1}, \ldots, f_{l}\right)$.

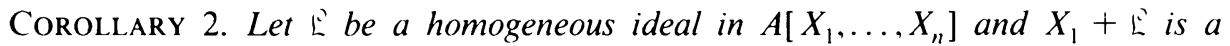
regular element in $A\left[X_{1}, \ldots, X_{n}\right] / E$. Then the number of minimal generators of $E^{\prime}=$ number of minimal generators of $\left(E^{\prime}, X_{1}\right) /\left(X_{1}\right)$.

Proof. $(\geqslant)$ It is obvious.

$(\leqslant)$ Suppose $\left\{f_{1}, \ldots, f_{t}\right\}$ is a subset of $E^{2}$ such that

$$
\left(f_{1}, \ldots, f_{t}\right)+\left(X_{1}\right) /\left(X_{1}\right)=\mathbb{E}+\left(X_{1}\right) /\left(X_{1}\right) \simeq E /\left(X_{1}\right) \cap E=E / X_{1} E,
$$

since $X_{1}+\mathbb{E}$ is a regular element in $A\left[X_{1}, \ldots, X_{n}\right] / \mathbb{E}$. Now, images of $f_{i}$ in $\mathbb{E} / X_{1} \mathbb{E}$ generate $\mathfrak{L}^{\prime} / X_{1} \mathfrak{L}^{\prime}$. By Lemma $4, f_{1}, \ldots, f_{t}$ generate $\mathfrak{L}^{\prime}$.

THEOREM 2. Let $(R, \mathrm{II})$ be a d-dimensional Cohen-Macaulay local ring and $I$ be an II-primary ideal such that $\operatorname{depth} G_{I}(R) \geqslant d-1$ and $G_{I}(R)$ is free over $R / I$. Then $M \leqslant e(R)$.

Proof. By Lemmas 2 and 3, we just need to prove the theorem in the ring $R(u)$. Therefore we can assume $R / \mathrm{m}$ is infinite. We will prove the theorem by induction on $d$. When $d=1$, we showed it in Corollary 1. Suppose $d>1$. First, we can choose $x_{1} \in I \backslash I^{2}$ such that $\bar{x}_{1}$ is a regular element in $G_{I}(R)$. (Sketch of proof: Let Ass $G_{I}(R)=\left\{p_{1}, \ldots, p_{t}\right\}$. Then

$$
p_{i} \cap I / I^{2} \subsetneq I / I^{2}, \quad \text { for any } i=1, \ldots, t .
$$

Since $R / \mathrm{m}$ is infinite, $I / I^{2} \supsetneqq \cup_{i=1}^{t}\left(p_{i} \cap I / I^{2}\right)$. Choose $x_{1} \in I \backslash I^{2}$ such that $\bar{x}_{1} \in I / I^{2} \backslash \cup_{i=1}^{t}\left(p_{i} \cap I / I^{2}\right)$.) Note that such $x_{1}$ is a nonzero divisor in $R$. We can check that $G_{I / x_{1} R}\left(R / x_{1} R\right) \simeq G_{I}(R) / \bar{x}_{1} G_{I}(R)$, and $R / x_{1} R$ is a CohenMacaulay local ring of dimension $d-1$ and $\operatorname{depth} G_{I / x_{1} R}\left(R / x_{1} R\right) \geqslant d-2$, and $\operatorname{dim}_{k}\left(I / I \mathrm{~m}+x_{1} R\right)=\operatorname{dim}_{k}(I / I \mathrm{~m})-1$, and $e\left(R / x_{1} R\right)=e(R)$. (See [5] for the reference.) Therefore we can assume that the theorem is true for $R / x_{1} R$ by the 
induction hypothesis. Next, choose $x_{2}, \ldots, x_{l}\left(l=\operatorname{dim}_{h}(I / I \| \mathrm{I})\right)$ such that $\left\{\bar{x}_{i}\right\}_{i} \quad 1, \ldots l$ is a minimal basis for $I / I^{2}$. Define $\phi: R / I\left[X_{1}, \ldots, X_{l}\right] \rightarrow G_{I}(R)$ by $\phi\left(X_{i}\right)=\bar{x}_{i}$ for any $i=1, \ldots, l$. Let $E=$ Kernel $\phi$ and $f_{1}, \ldots, f_{t}$ be homogeneous elements which form a minimal basis of $E^{\prime}$. Then

$$
\begin{aligned}
G_{I / x_{1} R}\left(R / x_{1} R\right) & \simeq G_{I}(R) / \bar{x}_{1} G_{I}(R) \simeq \frac{R / I\left[X_{1}, \ldots, X_{l}\right] / \mathfrak{L}}{X_{1}\left(R / I\left[X_{1}, \ldots, X_{l}\right] / \mathcal{L}\right)} \\
& \simeq \frac{R / I\left[X_{1}, \ldots, X_{l}\right]}{\left(X_{1}\right)+\varrho} \simeq \frac{R / I\left[X_{2}, \ldots, X_{l}\right]}{\left(f_{1}, \ldots, f_{t}\right)+\left(X_{1}\right) /\left(X_{1}\right)} .
\end{aligned}
$$

By assumption, there exists a minimal set $g_{1}, \ldots, g_{s}$ such that $g_{i}$ 's are homogeneous elements with respect to $X_{2}, \ldots, X_{1}$ and $\operatorname{deg} g_{i} \leqslant e(R)$ for any $i$, and

$$
\left(f_{1}, \ldots, f_{t}\right)+\left(X_{1}\right) /\left(X_{1}\right)=\left(g_{1}, \ldots, g_{s}\right)+\left(X_{1}\right) /\left(X_{1}\right) \text {. }
$$

We will show that $\max \operatorname{deg}_{1 \leqslant i \leqslant t} f_{i} \leqslant e(R)$. Suppose not, i.e. $\operatorname{deg} f_{1}>e(R)$. Then $g_{i} \in\left(f_{2}, \ldots, f_{t}\right)+\left(X_{1}\right)$ for any $i=1, \ldots, s$, since $\operatorname{deg} g_{i} \leqslant e(R)$. Therefore $\left(g_{1}, \ldots, g_{s}\right)+\left(X_{1}\right)=\left(f_{2}, \ldots, f_{t}\right)+\left(X_{1}\right)$ so that $\left(f_{1}, \ldots, f_{t}\right)+\left(X_{1}\right) /\left(X_{1}\right)=$ $\left(f_{2}, \ldots, f_{t}\right)+\left(X_{1}\right) /\left(X_{1}\right)$. This contradicts that $\left\{\bar{f}_{1}, \ldots, \bar{f}_{t}\right\}$ is a minimal basis of

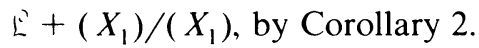

COROllary 3. Let $(R, m)$ be a d-dimensional Cohen-Macaulay local ring such that $\operatorname{depth} G_{\mathrm{m}}(R) \geqslant d-1$. Assume that $R=S / J$ with $(S, \tilde{\mathrm{m}})$ an l-dimensional regular local ring and $J \subset \tilde{\mathrm{m}}^{2}$. Then $G_{\mathrm{m}}(R) \simeq k\left[X_{1}, \ldots, X_{l}\right] / \mathcal{L}, k=S / \tilde{\mathrm{m}}$, where the degrees of elements in a homogeneous minimal basis of $\mathcal{L}$ are at most $e(R)$.

REMARK 2. In many cases max $\operatorname{deg}_{1 \leqslant i \leqslant t} f_{i}$ may be strictly less then $r(I)+1$. (Cf. Theorem 1.) Consider $R=k\left[\left[t^{6} t^{7} t^{8}\right]\right], k$ is any field. Then $e(R)=6, r(\mathrm{~m})=3$, $G_{\mathrm{m}}(R) \simeq k[X, Y, Z] /\left(Z^{3}, Y^{2}-X Z\right)$. Hence the maximal degree of generators < $r(\mathrm{~m})+1$. But, consider $R=k\left[\left[t^{6}, t^{7}, t^{15}\right]\right]$. Then $e(R)=6, r(\mathrm{~m})=5, G_{\mathrm{m}}(R) \simeq$ $k[X, Y, Z] /\left(X Z, Z^{2}, Z Y^{3}, Y^{6}\right)$. Therefore, in this case the maximal degree of generators $=r(\mathrm{~m})+1$.

This note forms a part of my doctoral thesis. I gratefully acknowledge my appreciation to Professor Judith Sally.

\section{REFERENCES}

1. H. Hironaka, Resolution of singularities of an algebraic variety over a field of characteristic zero. II, Ann. of Math. (2) 79 (1964), 205-326.

2. J. Herzog and R. Waldi, $A$ note on the Hilbert function of a 1-dimensional Cohen-Macaulay ring, Manuscripta Math. 16 (1975), 251-260.

3. H. Matsumura, Commutative algebra, 2nd ed., Benjamin Cummings, New York, 1980.

4. L. J. Ratliff, Jr., On quasi-unmixed local ciomains, the altitude formula, and the chain condition for prime ideals. II, Amer. J. Math. 92 (1970), 99-144.

5. J. D. Sally. Numbers of generators of ideals in local rings, Lecture Notes in Pure and Appl. Math., Dekker, New York and Basel, 1978.

6. O. Zariski and P. Samuel, Commutative algebra, Lecture Notes in Math., vol. 2, Springer-Verlag, Berlin and New York, 1975.

Department of Mathematics, Seoul National University, Seoul, Korea 